\title{
Ultrastructural Visualization of Complex Carbohydrates in Odontoblasts, Predentin, and Dentin Matrix by the Tannic Acid-Uranyl Acetate Method
}

\author{
by \\ Masato KAGEYAMA, Hidehito KADO, Noboru KAJIYAMA, \\ Kunihito KADO, Ken NAGAI, Masaki FUJITA, \\ and Yoshio KASHIWAYA
}

(Received 5 September 1985)

\section{Introduction}

It has been shown previously by histochemical, biochemical, and autoradiographic methods that complex carbohydrates such as glycoproteins and proteoglycans are complex deeply involved in the formation of dentin ${ }^{[1,2]}$. The procedures used to detect these carbohydrates by electron-microscopic histochemical analysis are the phosphotungstic acid method ${ }^{[3,4,5]}$, the silver methenamine method ${ }^{[3]}$, the periodatethiocarbohydrazide-silver proteinate (PA-TCH-SP) method $^{[6]}$, the ruthenium red method $^{[4,7]}$, the Alcian blue method ${ }^{[5]}$, the bismuth nitrate method ${ }^{[4,5]}$, the colloidal thorium method ${ }^{[8]}$, the high iron diamine (HID-TCH-SP) method ${ }^{[6]}$, and the cuprolinic blue method ${ }^{[9]}$. Most of these methods, however, have various drawbacks ${ }^{[6]}$, so that there are in actuality few appropriate methods for the accurate detection of the complex carbohydrates involved in dentin formation.

In a study on the epiphyseal cartilage of the tibia in rats, TAKAGI et al. ${ }^{[10]}$ used the tannic acid-uranyl acetate method ${ }^{[11]}$ (hereafter referred to as the TA-UA method), a method for the detection of complex carbohydrates that can be used with ultrathin sections, and reported that this method is useful for the staining of glycogen and glycoproteins, as well as proteoglycans. In addition, KAGEYAMA ${ }^{[12]}$ investigated staining by the TA-UA method in the auricular cartilage in rabbits and the proximal end of the diaphysis of the tibia in rats and obtained results that corresponded to those of TAKAGI et $\mathrm{al}^{[10]}$. He reported that the TA-UA method was an effective electron-microscopic histochemical procedure for the detection of complex carbohydrates.

We therefore used the TA-UA method to observe ultrastructural localization of complex carbohydrates in the rat odontoblast, predentin, and dentin matrix, which have not previously been observed by this procedure. Our findings are presented in this report.

\section{Materials and Methods}

Sprague-Dawley rats of approximately $150 \mathrm{~g}$ each in body weight were used. 景山正登, 角 秀人, 梶山 惕, 角 邦人, 永井 謙, 藤田正樹, 柏谷良雄: Department of Anatomy, Nihon University School of Dentistry. 
The upper incisors were removed under Nembutal anesthesia. Each specimen was fixed for 3 hours in $2.7 \%$ glutaraldehyde buffered with $0.1 \mathrm{M}$ cacodylate buffer (pH 7.3) and then decalcified for 2 weeks in $10 \%$ ethylenediaminetetraacetic acid (EDTA) buffered with $0.1 \mathrm{M}$ cacodylate buffer ( $\mathrm{pH}$ 7.3). Then, after each specimen had been washed with $0.1 \mathrm{M}$ cacodylate buffer containing $7 \%$ sucrose $(\mathrm{pH} 7.3)$, it was dehydrated in an alcohol series without osmium fixation and embedded in Spurr's resin. Ultra-thin sections were cut with a Porter-Blum MT-5000 ultramicrotome, transferred to stainless steel grids, and stained using the procedure described below.

TA-UA method ${ }^{[10,11,12]}$ : The ultra-thin sections were immersed for 10 minutes in a fresh TA aqueous solution made by dissolving $5 \mathrm{~g}$ of TA (tannic acid, J.T. Baker Chemical Co., Phillipsburg, N.J., U.S.A.), in $95 \mathrm{ml}$ of distilled water. Then, the sections were washed with distilled water and immersed for 5 minutes in a fresh UA aqueous solution made by dissolving $1 \mathrm{~g}$ of UA (uranyl acetate, E. Merck, Darmstadt, West Germany) in $99 \mathrm{ml}$ of distilled water. After washing with distilled water, the sections were observed using a transmission electron microscope at an accelerating voltage of $75 \mathrm{kV}$. Sections that were not stained by the TA-UA method were used as controls.

\section{Results}

Because there have been previous reports of electron-microscopic studies on the morphology of odontoblasts, predentin, and dentin ${ }^{[3,13-16]}$, the present report will deal only with the morphological findings necessary to interpret the results of TA-UA staining. The odontoblasts contained an abundance of rough endoplasmic reticulum and a well-developed Golgi apparatus with flat and partly distended cisternae. Secretory granules in various stages of maturation, lysosome-like granules with uniform contents, multi-vesicular bodies and tubulo-vesicular structures were observed in the Golgi region.

By the TA-UA method, there was weak staining of the distended portion of the Golgi cisternae in the odontoblasts (Fig. 1), while, among the rod-shaped secretory granules in various stages of maturation, there was moderate staining of the immature secretory granules proximal to the mature face of the Golgi cisternae (Fig. 1) and intense staining of the mature secretory granules located in the odontoblast process or at its basal portion (Figs. 1,3). The multi-vesicular bodies located in the Golgi region showed moderate staining in their matrix. Moderate staining was also observed in the surrounding tubulo-vesicular structures (Fig. 1). The spherical lysosomal granules with uniform contents showed weak TA-UA staining (Fig. 2). The nucleus, the rough endoplasmic reticulum, and mitochondria were not stained by TA-UA (Figs. 1,3). The collagen fibrils in the predentin were thinner (approximately $50 \mathrm{~nm}$ ) than those in the calcified dentin. They were arranged comparatively randomly in irregularly-oriented directions and demonstrated moderate TAUA staining (Fig. 3). In addition, the small granular substances scattered among these collagen fibrils showed very weak TA-UA staining (Fig. 4). As the predentin changed to calcified dentin, the diameter of the collagen fibrils increased (approximately $100 \mathrm{~nm}$ ). The collagen fibrils became arranged more densely and became intensely stained by the TA-UA method (Figs. 3, 5). There was intense TA-UA 


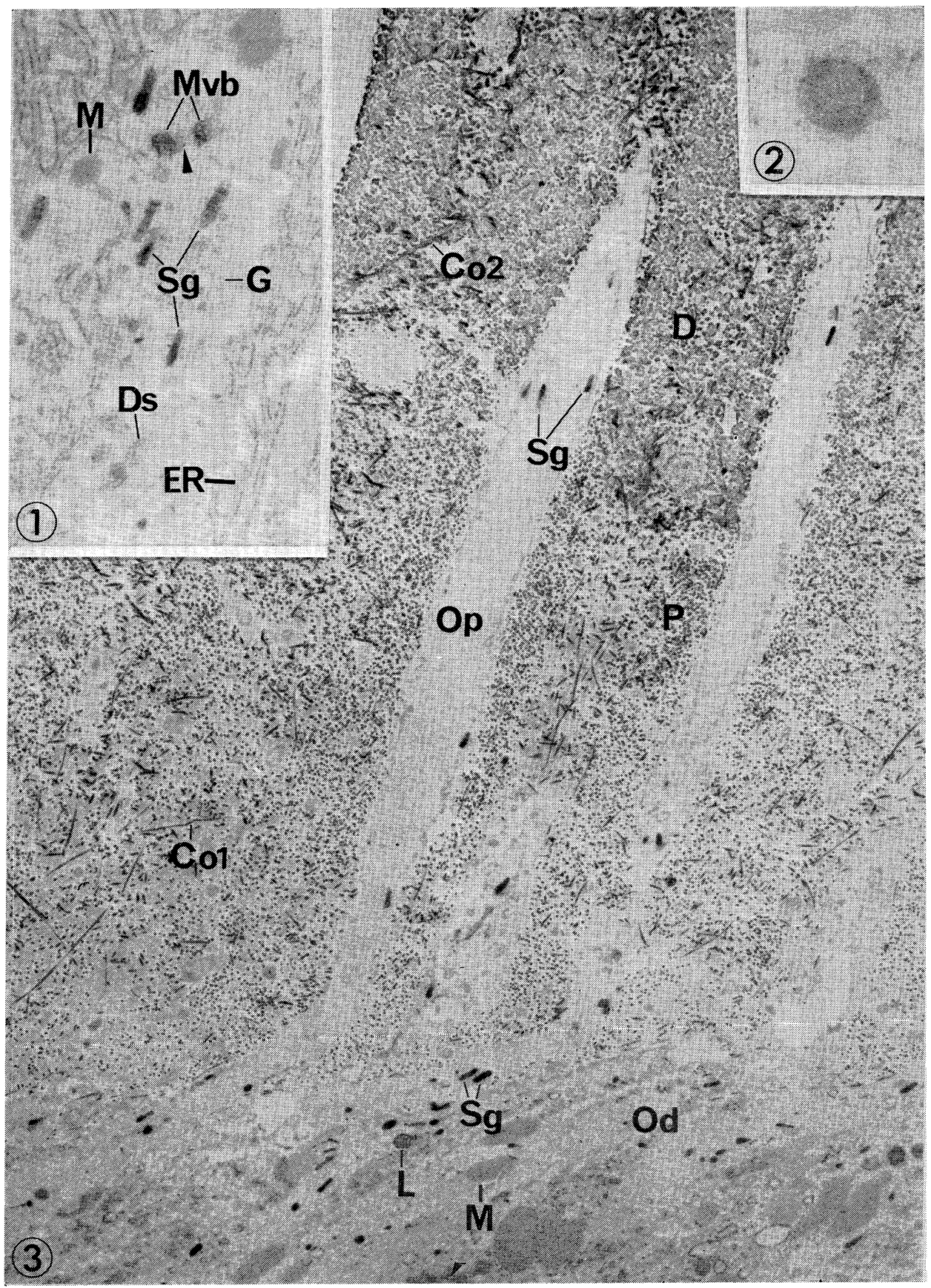



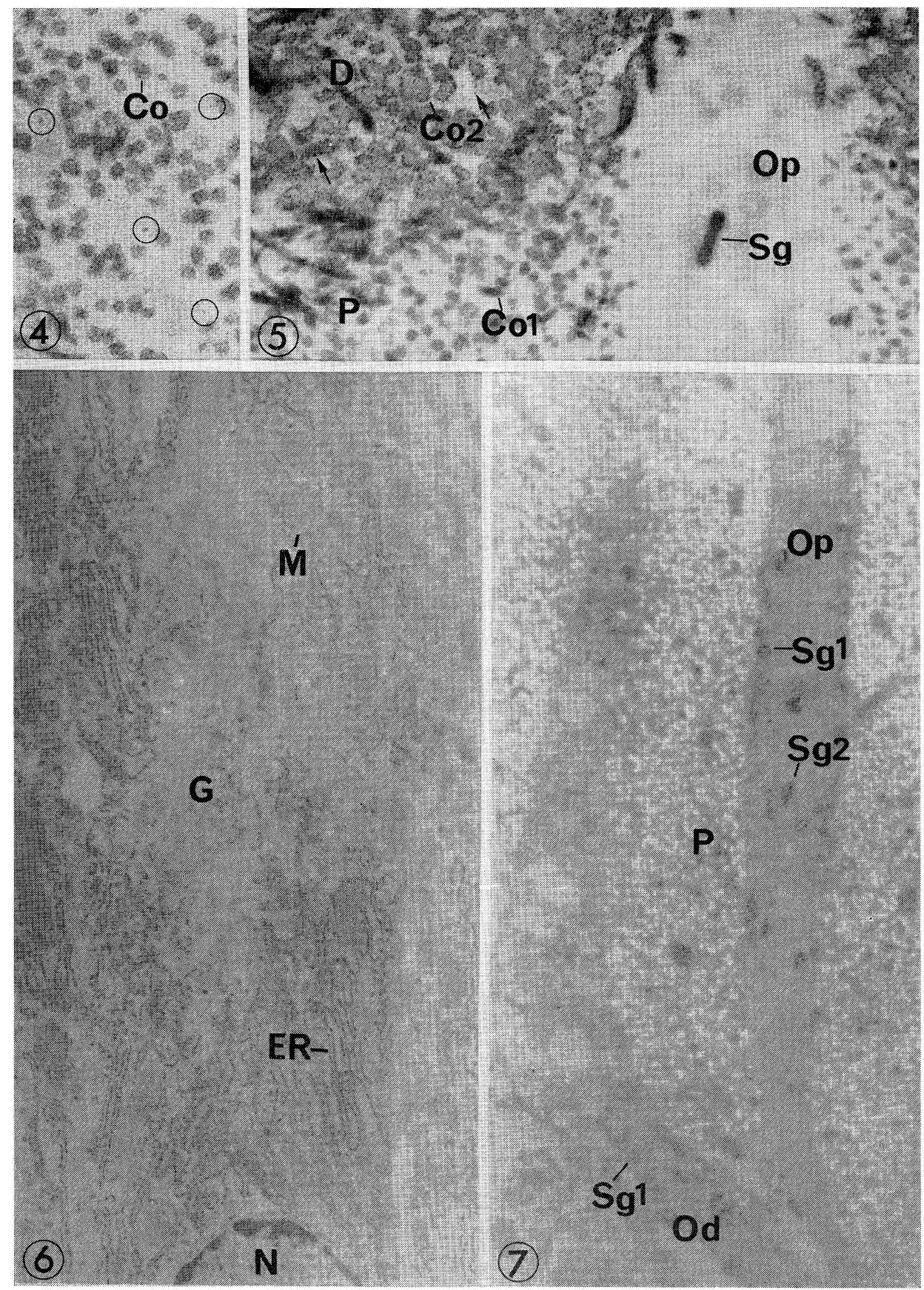
staining of the granular, non-collagenous substances existing between or appearing to be attached to the collagen fibrils (Fig. 5). In control specimens, no staining of the nucleus, rough endoplasmic reticulum, mitochondria, Golgi region, or collagen fibrils was observed (Fig. 6). However, slightly electron-dense material did exist in some secretory granules at the base of the odontoblast process (Fig. 7).

\section{Discussion and Conclusion}

We observed complex carbohydrates in rat odontoblasts, predentin, and dentin matrix using the TA-UA method and investigated their staining and localization. Complex carbohydrates fall into two categories, polysaccharides and glycoconjugates, the former being further subdivided into homopolysaccharides and heteropolysaccharides and the latter into glycoproteins, proteoglycans, and glycolipids ${ }^{[17]}$. Previous ultrastructural studies ${ }^{[10,12]}$ of epiphyseal cartilage, elastic cartilage, and bone tissue have shown the TA-UA method to be specific for glycogen, glycoproteins, and proteoglycans.

The rat odontoblast synthesizes glycoproteins and proteoglycans in the Golgi apparatus, transports most of these products to secretory granules, and also appears to secrete them into the extracellular matrix ${ }^{[1,2,6,18-20]}$. In addition, it is known that some glycoprotein in the odontoblasts is localized in the lysosomes, multi-vesicular bodies, and tubulo-vesicular structures ${ }^{[3,6]}$. The findings obtained in the present study using TA-UA staining closely corresponded with those obtained by the PATCH-SP and HID-TCH-SP methods, which are used in the electron-microscopic

Fig. 1 Golgi region of an odontoblast after staining by the TA-UA method. There is weak TA-UA staining of the distended portion (Ds) of the Golgi cisternae and moderate staining of the immature secretory granules $(\mathrm{Sg})$, the matrix of the multi-vesicular bodies $(\mathrm{MVb})$, and the tubulo-vesicular structures (arrow). However, there is no TA-UA staining of intracellular organelles such as the rough endoplasmic reticulum (ER) or mitochondria (M). Golgi apparatus: G. $\times 21,000$.

Fig. 2 Lysosomal bodies of odontoblasts stained weakly by the TA-UA method. $\times 39,000$.

Fig. 3 Low-power view of odontoblasts (OD), odontoblast processes (Op), and dentin matrix stained by the TA-UA method. The mature secretory granules $(\mathrm{Sg})$ at the base of, or within the odontoblast process show intense TA-UA staining. The collagen fibrils in the predentin (Col) show moderate staining. The collagen fibrils in the calcified dentin matrix (Co2) show intense TA-UA staining. Lysosomal bodies: L. Mitochondria: M. $\times 9,000$.

Fig. 4 High-power view of predentin stained by the TA-UA method. The small granular substances (inside circles) scattered among the collagen fibrils (Co) show weak staining. $\times 45,000$.

Fig. 5 High-power view of mineralization front of dentin matrix stained by the TA-UA method. The granular substances (arrow) existing between or appearing to be attached to the collagen fibrils (Co2) of the calcified dentin matrix show intense TA-UA staining. Predentin: P. Collagen fibrils in predentin: Col. Odontoblast process: Op. Mature secretory granules: Sg. $\times 30,000$.

Fig. 6 Odontoblasts not stained by the TA-UA method. No staining is seen in intracellular organelles such as the nucleus (n), Golgi region (G), rough endoplasmic reticulum (ER), or mitochondria $(\mathrm{M}) . \times 12,000$.

Fig. 7 The basal area of the odontoblast process (Od), the odontoblast process (Op), and predentin (P) without TA-UA staining. No staining is observed in the secretory granules (Sgl) or the collagen fibrils in the predentin or dentin matrix. However, there is a slightly electron-dense substance in a portion of the secretory granules $(\mathrm{Sg} 2) . \times 12,000$. 
detection of the complex carbohydrates involved in dentin formation ${ }^{[6]}$. In addition, because it has been reported that the HID-TCH-SP method, by which it is possible to detect sulfated proteoglycans, demonstrates a substance attached to or situated between the collagen fibrils in predentin ${ }^{[6]}$, we believe that the small granular substances scattered between the collagen fibrils in the present study, which show extremely weak TA-UA staining (Fig. 4), correspond to proteoglycans.

The intracellular and extracellular ultrastructural localization of complex carbohydrates involved in dentin formation has been studied by various methods. Of these, it is possible to omit osmium post-fixation with the HID-TCH-SP method ${ }^{[6]}$, PATCH-SP method ${ }^{[6]}$, and the silver methenamine method ${ }^{[3]}$. However, with the ruthenium red method ${ }^{[4]}$, Alcian blue method ${ }^{[5]}$, bismuth nitrate method ${ }^{[4,5]}$, and the phosphotungstic acid method ${ }^{[3,4,5]}$, post-fixation with lipophilic osmium tetroxide must be performed when observing the reaction products by electron microscopy. In addition, of the reagents used in these studies, ruthenium red cannot generally pass through the cell membrane because it is a macromolecular substance. Therefore, it is impossible to detect intracellular substances that would be stained by ruthenium red ${ }^{[7]}$. Meanwhile, it is known that the secretory granules in odontoblasts are stained by the Alcian blue method ${ }^{[5]}$, the bismuth nitrate method ${ }^{[4,5]}$, and the phosphotungstic acid method ${ }^{[3,4,5]}$. However, because the secretory granules themselves have an essential affinity to osmium ${ }^{[6]}$, it is impossible to differentiate them from the complex carbohydrates stained by these methods. For these reasons, in contrast to the Alcian blue method ${ }^{[5]}$, bismuth nitrate method ${ }^{[4,5]}$, and phosphotungstic acid method $^{[3,4,5]}$, the TA-UA method, which does not require osmium fixation, facilitates specific staining of the complex carbohydrates of the secretory granules.

The non-collagenous granular substances existing between or appearing to be attached to the collagen fibrils at the mineralization front of dentin matrix ${ }^{[1,2,16,21]}$ showed intense TA-UA staining in the present study (Figs. 3, 5). This finding was very similar to that obtained for the same site by the PA-TCH-SP method, which specifically detects glycoproteins ${ }^{[6]}$. In addition, because it has been reported that proteoglycans decrease suddenly at the mineralization front ${ }^{[6]}$, we believe that the non-collagenous granular substances localized in the mineralization front revealed by the TA-UA method appear to be glycoproteins.

\section{References}

[1] Weinstock, A.: Elaboration of enamel and dentine matrix glycoproteins. In The Biochemistry and Physiology of Bone, Vol. 2, 121-154, Bourne, G. H., Ed., Academic Press, New York, 1972

[2] Leblond, C. P. \& Weinstock, M.: A comparative study of dentin and bone formation. In The Biochemistry and Physiology of Bone, Vol. 4, 517-562, Bourne, G. H., Ed., Academic Press, New York, 1976

[3] Weinstock, M. \& Leblond, C. P.: Synthesis, migration, and release of precursor collagen by odontoblasts as visualized by radioautography after $\left({ }^{3} \mathrm{H}\right)$ proline administration, J. Cell Biol., 60, 92-127, 1974

[4] Nygren, H., Hansson, H. A. \& Linde, A.: Ultrastructural localization of proteoglycans in the odontoblast-predentin region of rat incisor, Cell Tissue Res., 168, 277-287, 1976

[5] Goldberg, M., Genotelle-Septier, D. \& Weill, R.: Glycoprotéines et protéoglycans dans la 
matrice prédentinaire et dentinaire chez le rat: une étude ultrastructurale, J. Biol. Buccale, 6 , 75-90, 1978

[6] Takagi, M., Parmley, R. T. \& Denys, F. R.: Ultrastructural localization of complex carbohydrates in odontoblasts, predentin, and dentin, J. Histochem. Cytochem., 29, 747-758, 1981

[7] Nagai, N., Takuma, S., Goto, Y. \& Ogiwara, H.: Electron microscopy of dentine and predentine of developing rat molars stained with ruthenium red, J. Biol. Buccale, 2, 73-83, 1974

[8] Bevelander, G. \& Nakahara, H.: The formation and mineralization of dentin, Anat. Rec., 156, 303-323, 1966

[9] Goldberg, M. \& SePtier, D.: Electron microscopic visualization of proteoglycans in rat incisor predentine and dentine with cuprolinic blue, Archs. oral Biol., 28, 79-83, 1983

[10] Takagi, M., Parmley, R. T., Denys, F. R. \& Kageyama, M.: Ultrastructural visualization of complex carbohydrates in epiphyseal cartilage with the tannic acid-metal salt method, $J$. Histochem. Cytochem., 31, 783-790, 1983

[11] Sannes, P. L., Katsuyama, T. \& Spicer, S. S.: Tannic acid-metal salt sequences for light and electron microscopic localization of complex carbohydrates. J. Histochem. Cytochem., 26, 55-61, 1978

[12] Kageyama, M.: Ultrastructural cytochemistry of complex carbohydrates in auricular cartilage and bone tissue with the tannic acid-uranyl acetate method, Jpn. J. Oral Biol., 25, 104-115, 1983

[13] Garant, P. R., Szabo, G. \& Nalbandian, J.: The fine structure of the mouse odontoblast, Archs. oral Biol., 13, 857-876, 1968

[14] Reith, E. J.: Collagen formation in developing molar teeth of rats, J. Ultrastruct. Res., 21, $383-414,1968$

[15] Takuma, S. \& Nagai, N.: Ultrastructure of rat odontoblasts in various stages of development and maturation, Archs. Oral Biol., 16, 993-1011, 1971

[16] NaGAi, N.: Life cycle and function of odontoblast, The Cell, 10, 406-418, 1978

[17] Spicer, S. S., Schulte, B. A., Thomopoulos, G. N., Parmley, R. T. \& Takagi, M.: Cytochemistry of complex carbohydrates by light and electron microscopy: available methods and their application. In Connective Tissue Diseases, International Academy of Pathology Monograph, 163-211, Wagner, B. M., Fleischmajer, R. and Kaufman, N., Eds., Williams and Wilkins Co., Baltimore, 1983

[18] Lennox, D. W. \& Provenza, D. V.: Mucopolysaccharides in odontogenesis. Histochemical and autoradiographic study, Histochemie, 23, 328-341, 1970

[19] Sundström, B.: New aspects on the utilization of inorganic sulphate during dentin formation, Histocoemie, 26, 61-66, 1971

[20] Weinstock, A., Weinstock, M. \& Leblond, C. P.: Autoradiographic detection of ${ }^{3}$ H-fucose incorporation into glycoprotein by odontoblasts and its deposition at the site of the calcification front in dentin, Calcif. Tissue Res., 8, 181-189, 1972

[21] Weinstock, M. \& Leblond, C. P.: Radioautographic visualization of the deposition of a phosphoprotein at the mineralization front in the dentin of the rat incisor, J. Cell Biol., 56, $838-845,1973$ 